\title{
Erinnern! Auch wenn es unmöglich ist Mieczysław Weinbergs Oper Passažirka op. 97
}

\author{
Verena MogL \\ Institut für kulturelle Innovationsforschung an der \\ Hochschule für Musik und Theater Hamburg \\ Harvestehuder Weg 12, 20148 Hamburg, Deutschland \\ E-Mail: mogl.verena@gmail.com
}

(Angekommen: März 2016; angenommen: Juni 2016)

\begin{abstract}
In the search for reasons, why Weinberg's opera Passažirka op. 97 never made it into public despite the praise it received after various hearings, one reason can be found in the way the opera deals with remembrance, especially in the context of the Holocaust. Whereas remembrance provides a mental shelter to those in need, others are denied to take refuge in memories or are even forced to confront brutal truths. Not only the libretto but also the music deals with the act of remembrance in a way, that made the opera a daring composition in the time of its origin.
\end{abstract}

Keywords: Weinberg, opera, Holocaust

Im frühen Sommer 1968 vollendete der polnisch-jüdische Komponist Mieczysław Weinberg die Oper Passažirka op. 97, zu der Alexandr Medvedev das Libretto verfasst hatte. ${ }^{1}$ Der Oper, die seit ihrer szenischen Uraufführung im Sommer 2010 erfolgreich an zahlreichen Opernhäusern aufgeführt und von der internationalen Presse mit Lob bedacht wurde, ${ }^{2}$ maß auch der Komponist selbst die allergrößte Bedeutung zu. So antwortete Weinberg auf die Frage, welches seiner Werke ihm am meisten am Herzen liege:

1. Der vorliegende Aufsatz stützt sich maßgeblich auf Untersuchungen, die in das dritte Kapitel meiner Dissertationsschrift: , Juden, die ins Lied sich retten“ - Der Komponist Mieczysław Weinberg (1919-1996) in der Sowjetunion (Kassel: Waxmann, im Druck) einflossen.

2. So gab es nach Bregenz u. a. gefeierte Aufführungen in London (2011), Warschau (2010), New York (2014) und Frankfurt (2015). Ich danke Herr Arnt Nitschke von Peermusic Classical für die freundliche Bereitstellung der gesammelten Presserezensionen. 
Das wichtigste [Werk - V. M.] - Das ist Passažirka. Alle übrigen sind auch Passažirka. Von den letzten Symphonien die 21., die bisher noch nicht aufgeführt wurde. Sie ist den im Warschauer Ghetto Verbrannten gewidmet, wo auch meine nächsten Angehörigen ermordet wurden. Diese Symphonie - ist auch die Passažirka. ${ }^{3}$

\section{Ein unerhörtes Meisterwerk}

Nicht nur, dass insgesamt kaum Selbstaussagen Weinbergs zu seiner Musik bekannt sind, darüber hinaus ist diese besondere Hervorhebung von Opus 97 auch angesichts des äußert vielgestaltigen und umfangreichen Werkkatalogs bemerkenswert: So umfasst das Euvre mehr als 150 Kompositionen mit Opusbezifferung - darunter u. a. 21 vollendete Symphonien, 4 (umfangreiche) Kammersymphonien, 17 Streichquartette und insgesamt 8 Opern und Operetten. ${ }^{4}$

Natürlich kann an dieser Stelle keine umfassende Analyse von Passažirka erfolgen. ${ }^{5}$ Jedoch soll ein besonderer Aspekt des Werks herausgegriffen und untersucht werden. Denn es stellt sich die Frage, weshalb dieses große Werk fast 40 Jahre auf seine konzertante, und über 40 Jahre auf seine szenische Uraufführung warten musste. ${ }^{6}$ Und dies, obwohl das Werk nach Anhörung(en) im Moskauer Komponistenverband, die kurz nach dessen Fertigstellung stattgefunden hatte(n), ${ }^{7}$ wie auch bei einer Darbietung im privaten $\mathrm{Kreis}^{8}$ von zahlreichen Kollegen in den höchsten Tönen gelobt worden war. ${ }^{9}$ Sogar Tichon Chrennikov, der langjährige Vorsitzende des Sowjetischen Komponistenverbandes, erwähnte 1969 auf dem vierten Verbandskongress (der kurz nach einer der Anhörungen der Oper stattgefunden hatte) Passažirka als ein Beispiel für eine gelungene Opernkomposition, deren Aufführung ,wünschenswert“ wäre:

3. Ljudmila Nikitina, Simfonii M. Vajnberga (Moskau: Izd. Muzyka, 1972), 23. Übertragung aus dem Russischen - V. M.

4. Vgl. dazu das Werkverzeichnis in meinem Artikel „Mieczysław Weinberg“, in Komponisten der Gegenwart, hrsg. von Hanns-Werner Heister und Walter-Wolfgang Sparrer (München: Edition Text + Kritik, 2012), 47. Nlfg., W-Z7.

5. Neben Mogl, ,Juden, die ins Lied sich retten“, v. a. Abschnitt 3, setzt sich in einer aktuellen Publikation auch Wolfgang Mende mit der Passagierin auseinander: Wolfgang Mende, ,Sozrealismus als kulturelles Regulativ. Weinbergs Opern Die Passagierin und Die Madonna und der Soldat", Tonkunst 2/10 (April 2016), 135-149, darin v. a. 139-144.

6. Die konzertante Uraufführung erfolgte 2006 in Moskau, die szenische Uraufführung erfolgte $2010 \mathrm{im}$ Rahmen der Bregenzer Festspiele.

7. Eine Anhörung im Komponistenverband fand statt am 1. November 1968, vgl. RGALI, f. 2490, op. 2, ed. chr. 191: Stenogramma zasedanija Sekretariata po obsuždeniju opery M. S. Vajnberga „Passažirka“. In diesem Stenogramm erwähnen mehrere Teilnehmer, die Oper nun schon zum zweiten Mal gehört zu haben. Ob es sich dabei um die unten erwähnte private Aufführung handelt, ist nicht geklärt.

8. Auf der oben genannten Anhörung berichtete Dmitrij Šostakovič, das Werk bereits auf einer privaten Darbietung gehört zu haben; vgl. RGALI, Stenogramma, 1. 5.

9. Unter anderem von Dmitrij Šostakovič, Rodion Ščedrin oder Dmitrij Kabalevskij, vgl. RGALI, Stenogramma, 1. 1. 
Vor kürzester Zeit wurden einige neue Opern beendet, die noch nicht aufgeführt wurden - Passažirka von M. Weinberg, Sestry von D. Kabalevskij sowie neue Kompositionen [...]. Es ist noch zu früh, um sie [die Kompositionen V. M.] erschöpfend zu bewerten, aber allein der Fakt, dass das Interesse am Operngenre gewachsen ist, muss uns erfreuen. Die schnellstmögliche szenische Aufführung der besten Opern-Neuheiten ist wünschenswert. ${ }^{10}$

Angesichts dieser Umstände ist umso verwunderlicher, weshalb die von allen Seiten hochgelobte Oper erst zehn Jahre nach Weinbergs Tod (1996) zur ersten Aufführung gelangte.

Um zur Klärung dieser Frage beizutragen, ist es in einem ersten Schritt notwendig, auch einige Worte zum biographischen Hintergrund Weinbergs sowie zu einigen entscheidenden Punkten innerhalb seines Karriereverlaufs zu verlieren. Denn wie bereits an anderer Stelle ausführlich dargelegt werden konnte, ${ }^{11}$ waren hinsichtlich der Frage, ob Weinbergs Werke die zensorischen Hürden überwinden konnten, nicht nur konkrete Charakteristika der Kompositionen an sich, sondern darüber hinaus auch bestimmte biographische Faktoren von größter Bedeutung. Im Zusammenhang damit ist es - um Passažirka richtig einordnen zu können weiterhin unerlässlich, sich verschiedene Schaffensphasen, die Weinberg durchlief, zu vergegenwärtigen.

\section{Zur Biographie Weinbergs}

Weinberg wurde am 8. Dezember 1919 in Warschau geboren. Die Familie war tief in der jüdischen Gemeinde Warschaus verwurzelt und beide Elternteile waren lange Jahre aktive Mitglieder in verschiedenen jüdischen Theatertruppen gewesen. ${ }^{12}$ Mit dem Überfall der Nationalsozialisten auf Polen im Herbst 1939 sah sich Weinberg, der in Warschau bereits erfolgreich Klavier studiert hatte, als junger Mann zur Flucht gezwungen. Nach Stationen in Minsk - dort absolvierte er ein Kompositionsstudium - und weiterhin Taschkent gelangte er im Jahr 1943 nach Moskau, wo er bis zu seinem Lebensende im Februar 1996 lebte und arbeitete. ${ }^{13}$ Seine Mutter, sein Vater und seine jüngere Schwester, die in Polen zurückgeblieben waren, ${ }^{14}$ überlebten den Holocaust nicht.

10. Tichon Chrennikov, „[Četvertyj s”ezd Sojuza kompozitorov SSSR]“, Sovetskaja muzyka 2 (1969), 4-18, hier: 11f.

11. Vgl. dazu meine Ausführungen in Mogl, „Juden, die ins Lied sich retten“, [Kapitel 1].

12. Vgl. Mogl, „Juden, die ins Lied sich retten“, Kapitel 1.

13. Zur Biographie Weinbergs nach der Ankunft in Moskau vgl. auch bei David Fanning, Mieczysław Weinberg. Auf der Suche nach Freiheit (Hofheim: Wolke, 2010).

14. Bisher gibt es keine genauen Angaben zu einer eventuellen Flucht von Weinbergs Familie aus Polen und zu den Umständen ihres Todes. 
In Moskau gelang es Weinberg schnell, sich als aufstrebender Komponist einen Namen zu machen. Er wurde in den Komponistenverband aufgenommen, bedeutende Interpreten wie David Oistrach oder Ėmil' Gilel's spielten seine Musik und prominente Kollegen wie Nikolaj Mjaskovskij und Dmitrij Šostakovič setzten sich für ihn und seine Werke ein. ${ }^{15}$ Zwischen Šostakovič und Weinberg entwickelte sich schnell eine enge Freundschaft, die bis zum Tode Šostakovičs im August 1975 anhielt.

Schon bald nach Weinbergs Ankunft in Moskau jedoch wurde das zunehmend repressive Klima des stalinistischen Regimes auch für den Komponisten spürbar. Der wachsende Druck, der zunächst in beruflicher, dann auch in privater Hinsicht auf Weinberg ausgeübt wurde, war einerseits seiner durchaus ambitionierten $\mathrm{Mu}$ siksprache und der Nähe zu von politischer Seite mit Argwohn bedachten Komponistenkollegen - wie etwa Dmitrij Šostakovič - geschuldet. Jedoch vor allem Weinbergs polnisch-jüdische Herkunft und seine enge familiäre Bindung zu dem prominenten Solomon Michoèls ${ }^{16}$ - Weinberg hatte im Taschkenter Exil dessen Tochter Natal'ja Vovsi-Michoèls geheiratet - brachten den Komponisten schließlich in höchste Bedrängnis. ${ }^{17}$ Nach langen Jahren der permanenten Überwachung und einer Reihe von (beruflichen) Schikanen wurde Weinberg schließlich im Februar 1953 inhaftiert. Nur dem plötzlichen Tod Stalins und der Fürsprache von Šostakovič war es zu verdanken, dass er noch im April desselben Jahres aus der Haft entlassen wurde. ${ }^{18}$

\section{Jahre der Rehabilitation - eine neue Schaffensphase}

In den Jahren, in denen der größte Druck auf Weinberg ausgeübt wurde (ungefähr zwischen 1948 und 1953) geriet seine Karriere merklich ins Stocken und sein kompositorischer Ertrag verringerte sich signifikant. ${ }^{19}$ Auch nach seiner Entlassung dauerte es mehrere Jahre, bis Weinberg zu voller Produktivität zurückfand.

Erst in den späten 1950er Jahren wurde der Komponist denn auch mehr oder weniger inoffiziell ,rehabilitiert ${ }^{6}{ }^{20}$ Dies geschah vor allem in Form von Werken, die vergleichsweise kurz nach ihrer Entstehung aufgeführt und/oder publiziert

15. So setzte sich Mjaskovskij unter anderem als Fürsprecher für das später verbotene 6. Streichquartett op. 35 ein; vgl. dazu die Anmerkungen in Nikolaj Ja. Mjaskovskij, Stat'i, pis'ma, vospominanija (Moskau: Sov. Kompozitor, 1960), Vol. 2, 242.

16. Solomon Michoèls * 16. März 1890, † Januar 1948, Schauspieler und Regisseur, ab 1942 Vorsitzender des Jüdischen Antifaschistischen Komitees.

17. Ein Überblick über die scharfen Kritiken und Verunglimpfungen, mit denen sich Weinberg konfrontiert sah, findet sich in Mogl, ,Juden, die ins Lied sich retten“, [Kapitel 1].

18. Ein Faksimile des Entlassungsformulars findet sich in Fanning, Auf der Suche nach Freiheit, 94.

19. Vgl. dazu die entsprechenden Zeitabschnitte im WVZ bei Mogl, Weinberg, K-Q.

20. Zu Formen der inoffiziellen Rehabilitierung in der Sowjetunion vgl. Albert van Goudoever, The Limits of Destalinization in the Soviet Union. Political Rehabilitations in the Soviet Union since Stalin (London Sydney: Croom Helm, 1986), v. a. 117ff. 
wurden. ${ }^{21}$ Und auch Werke, die bereits zu einem früheren Zeitpunkt komponiert worden waren, jedoch nie in der Öffentlichkeit aufgeführt wurden, wurden nun auf einmal zugänglich gemacht. ${ }^{22}$ Des Weiteren wurde Weinberg - dessen Name fast vollständig aus der Presse verschwunden gewesen war $^{23}$ - ein sehr ausführlicher und deutlich wohlwollender Artikel in der Fachpresse gewidmet. ${ }^{24}$ Diese ,inoffizielle Rehabilitierung' tat seine Wirkung: So ist aus der Retrospektive zu erkennen, dass etwa zur selben Zeit auch ein erneuter Anstieg von Weinbergs Produktivität zu verzeichnen ist. Darüber hinaus begann der Komponist, sich musikalisch mit einem neuen Themenkomplex zu beschäftigen.

Dieser Themenkomplex umfasste die Frage nach Weinbergs polnisch-jüdischer Existenz in der Sowjetunion, das Problem des Exils und die daraus folgende, schmerzhaft empfundene Ferne zur polnischen Heimat. Weiterhin verbunden war damit das Thema des permanenten Konflikts mit Antisemitismus und AntiPolonismus, mit dem sich Weinberg auch nach Stalins Tod in der Sowjetunion konfrontiert sah.

Die meisten Werke, in denen sich Weinberg mit diesen Themenbereichen auseinandersetzte, waren Vokalwerke nach Gedichten des polnisch-jüdischen Dichters Julian Tuwim. ${ }^{25}$ Und obwohl die meisten Kompositionen Weinbergs, die im Zeitraum zwischen ca. 1959 und 1966 entstanden, relativ schnell publiziert oder aufgeführt wurden, blieben just die Werke nach Texten von Tuwim fast ausschließlich in der so genannten, Schublade ${ }^{6}{ }^{26}$ Wie nachgewiesen werden konnte, war dies jedoch mehr den gewählten Texten - und damit den darin enthaltenen Themen - geschuldet, denn der Musik. ${ }^{27}$ Von insgesamt acht umfangreichen Werken aus dieser Zeit, die Weinberg nach Tuwim-Texten komponierte (zwei Vokalsymphonien, eine Kantate, vier Liederzyklen und ein alleinstehendes Lied), wurde allein die 8 . Symphonie öffentlich aufgeführt. ${ }^{28}$

21. So zum Beispiel die 2. Sinfonietta op. 74. Weinberg hatte sie Anfang Oktober 1960 komponiert und bereits am 19. November wurde sie uraufgeführt. Ähnlich verhielt es sich bei einer ganzen Reihe von Werken; vgl. dazu die entsprechenden Abschnitte bei Mogl, Weinberg, ab T ff.

22. So wurde beispielsweise das 4. Streichquartett op. 20, das Weinberg bereits 1945 komponiert hatte, erst 1959 erstmals verlegt: Moisej Vajnberg: Kvartet No. 4 dlja dvuch skripok, al'ta i violončeli, soč. 20 (Moskau: Sov. Kompozitor, 1959).

23. Und der in den Jahren der Repression vor allem diffamiert worden war; vgl. dazu Mogl, ,Juden, die ins Lied sich retten“, [Kapitel 1].

24. Aleksey Nikolaev, „O tvorčestve M. Vajnberga“, Sovetskaja Muzyka 1 (1960), 40-47.

25. Julian Tuwim, * 13. Sept. 1894, †27. Dez. 1953.

26. Vgl. dazu auch meinen Artikel „'Wenn's draußen neblig ist, fällt innen das Heimweh leichter, das Erinnern'“, Tonkunst 2/10 (April 2016), 172-181.

27. Vgl. dazu u. a. Mogl, ,Juden, die ins Lied sich retten“, [Kapitel 2] und Mogl, ,Wenn's draußen neblig ist“, $175 \mathrm{ff}$.

28. Die Liederzyklen op. 57 (1956), op. 62 (1957/58), op. 77 (1962) und op. 90 (1966), die Kantate Piotr Płaksin op. 91 (1966), die 8. Symphonie op. 82 (1964), die 9. Symphonie op. 93 und das Lied „O, siwa mgło“, op. 84 (1964). Der ebenfalls unaufgeführte Liederzyklus op. 4, den Weinberg nach Versen Tuwims komponierte, wird hier nicht angeführt, da er bereits 1940 komponiert wurde. Die Uraufführung der 8. Symphonie erfolgte am 6. März 1965 in Moskau. 


\section{Neue Strategien}

Weinberg jedoch war es offenkundig wichtig, sich trotz dieser Rückschläge mit den aufgeworfenen und offenkundig, schwierigen Themen zu beschäftigen, die in den ausgewählten Texten vor allem der Tuwim-Werke verhandelt wurden. Und naturgemäß muss ihm am Herzen gelegen haben, dass seine Werke auch Gehör fanden. So änderte der Komponist seine Herangehensweise: Zunächst wandte er sich nach Liedern und Vokalsymphonien einem anderen, einem , größeren' Genre zu, der Oper. Damit bediente Weinberg einerseits die von offizieller Seite stets gerühmte Vokalgattung, ${ }^{29}$ vermied jedoch gleichzeitig das ideologisch hochgradig aufgeladene Genre der Symphonie. ${ }^{30}$

Darüber hinaus widmete er sich einer Textvorlage, die sich in mehreren entscheidenden Punkten von seiner bisherigen literarischen Hauptquelle - dem dichterischen Nachlass Tuwims - unterschied. So diente nun die russische Übersetzung der Erzählung Pasażerka der ebenfalls polnischen, jedoch katholischen Autorin Zofia Posmysz als Grundlage für das Libretto. ${ }^{31}$ Während Tuwims Dichtungen $^{32}$ in der Sowjetunion durchaus kritisiert und auch zensiert worden waren und auch nicht vollständig in russischer Übertragung vorlagen, war die Erzählung Passažirka bereits bei ihrer ersten Publizierung in der Sowjetunion im Jahre 1963 ein großer Erfolg gewesen ${ }^{33}$ und in den Folgejahren gleich mehrmals wieder veröffentlicht worden. Hierin lag ein bedeutender Vorteil des Posmysz-Textes gegenüber den Tuwim-Dichtungen, dessen sich Weinberg sicherlich bewusst war.

In ihrem Text Die Passagierin erzählt Posmysz - die selbst in Auschwitz inhaftiert gewesen war - die Geschichte der ehemaligen Auschwitz-Aufseherin Anneliese Franz. Franz, die Jahre nach dem Krieg als Passagierin auf einem Überseedampfer nach Südamerika unterwegs ist, bemerkt an Deck eine andere Passagierin. In dieser Passagierin glaubt sie nun eine ehemalige Auschwitz-Inhaftierte zu erkennen, die im Lager ihrer Aufsicht unterstellt war. Die Handlung, die Posmysz daraufhin auf zwei verschiedenen Zeitebenen entwickelt - nach dem Krieg und während des Krieges - bietet eine neue, verstörende Perspektive auf die Grausamkeiten und Konflikte, die mit dem Konzentrationslager Auschwitz in Verbindung stehen. Nicht nur die Täterperspektive irritiert, zudem erfolgt in dem Text eine scharfe Ausleuchtung psychologischer Abgründe, die in ihrer Darstellung schockiert und aufwühlt.

29. Zur Bedeutung der Vokalgattung in der Sowjetunion vgl. Dorothea Redepenning, Geschichte der russischen und der sowjetischen Musik. Bd. 2: Das 20. Jahrhundert, Teilband 2 (Laaber: Laber Verlag, 2008), $510 \mathrm{ff}$.

30. Wie er es etwa in der 8. und 9. Symphonie getan hatte.

31. Polnische Erstveröffentlichung: Zofia Posmysz, Pasażerka (Warschau: Czytelnik, 1962).

32. Und auch der Dichter selbst; vgl. dazu die entsprechenden Abschnitte bei Mogl, „Juden, die ins Lied sich retten“, [Kap. 2].

33. Zof'ja Posmyš, „Passažirka“, Innostrannaja literatura 8 (August 1963), 4-87. Die Übersetzung aus dem Polnischen erfolgte durch Ė. Gessen und V. Golovskij. 
Die Arbeit am Libretto, ${ }^{34}$ die hauptsächlich Alexandr Medvedev übernahm, ${ }^{35}$ dauerte mehrere Jahre und erfolgte u. a. nach zwei Besuchen Medvedevs in Warschau bei der Autorin selbst. Im Zuge eines dieser Besuche reiste Medvedev zusammen mit Posmysz nach Auschwitz, um das ehemalige Lager zu besichtigen. ${ }^{36}$ Die Komposition stellte Weinberg innerhalb eines relativ kurzen Zeitabschnitts im Mai/Juni 1968 fertig.

\section{Die Oper Passažirka}

Insgesamt umfasst die Oper zwei Akte, acht Bilder und einen Epilog. Im Folgenden sollen in aller Kürze diejenigen Handlungsmomente kurz zusammengefasst werden, die hier von besonderer Bedeutung sind.

Die Handlung beginnt zeitlich nach dem Krieg und örtlich auf einem Überseedampfer Richtung Südamerika. Auf diesem Schiff sind der Deutsche Walter Kretschmer und seine junge Frau Lisa. Sie haben Deutschland verlassen und planen, sich in Südamerika niederzulassen. Auf dem Boot bemerkt Lisa plötzlich eine andere Passagierin und Lisa glaubt, diese Passagierin zu erkennen. Ein erster zeitlicher Rücksprung - der wie eine plötzlich aufflammende Erinnerung völlig unerwartet stattfindet - eröffnet daraufhin den Blick in eine andere zeitliche Ebene, in das Konzentrationslager Auschwitz, wo Lisa von einer Oberaufseherin für ihre Arbeit gelobt wird (Akt I, Bild 1, ab Zeichen 19). So wird dem Zuschauer klar, dass Lisa selbst Aufseherin in Auschwitz war. Wie sich dann herausstellt, hat sie diesen Teil ihrer Vergangenheit nicht nur ihrem Mann verschwiegen, sondern hat ihn auch selbst verdrängt. Ausgelöst durch dieses Moment bricht nun (zeitlich und örtlich wieder zurück auf der Ebene „Überseedampfer“) eine ganze Reihe von schrecklichen Erinnerungen über Lisa herein. Erinnerungen, die ihr keine Ruhe lassen. Als Walter Lisas Unruhe bemerkt und nach den Gründen dafür fragt, sieht sich Lisa schließlich gezwungen, ihrem Mann von ihrer Vergangenheit zu berichten und sich ihren Erinnerungen zu stellen.

An diesem Punkt wird im Libretto ein $\mathrm{Chor}^{37}$ in die Handlung eingeführt (Akt I, Bild 1, ab Zeichen 24), der im weiteren Verlauf der Oper - fast im Sinne eines griechischen Chores - die Handlung auf der Bühne von außen kommentiert und auch in die Handlung eingreift. Erst ausgelöst durch den mahnenden und drän-

34. Für Nachweise wird im Folgenden die deutsche Version des Librettos herangezogen: Mieczysław Weinberg: Die Passagierin. Libretto. Deutsche Adaption Ulrike Patow (Hamburg: Peermusic, 2010).

35. Anfänglich war offenbar auch ein gewisser Jurij Lukin in die Arbeit am Libretto involviert: In den ersten Versionen des Librettos ist sein Name explizit erwähnt. Jedoch ist die genaue Rolle Lukins bis heute ungeklärt und es kann festgehalten werden, dass hauptsächlich Medvedev an dem Libretto arbeitete.

36. Interview von Arnt Nitschke mit Zofia Posmysz im Mai 2014. Ich danke Herrn Nitschke für die Bereitstellung des Materials. Neben der Erzählung von Posmysz verarbeitete Medvedev auch noch Elemente zwei weiterer Romane in dem Libretto; vgl. dazu Mogl, Juden [v. a. Abschnitt 3].

37. Hinter der Bühne, besetzt mit Bässen. 
genden Gesang des Chores springt die Handlung vom Dampfer erneut zeitlich zurück in die Kriegsjahre, örtlich in das Konzentrationslager (ab dem 2. Bild). Auf der Bühne wird nun ausführlich eine Reihe von Situationen und Konflikten dargestellt, in welche Lisa und Martha - eine polnische Gefangene und die Frau, die Lisa auf dem Schiff erkannt zu haben glaubt - verwickelt waren. Es wird deutlich, dass Lisa in ihrer Funktion als Aufseherin Martha auf perfide Art und Weise zu instrumentalisieren versuchte, um dadurch mehr Macht auch über die anderen Gefangenen zu erlangen.

Die Handlung springt im Folgenden von der Ebene „Auschwitz“ immer wieder auf die Ebene „Überseedampfer“ zurück, wo Lisa verzweifelt versucht, sich ihrem schockierten Ehemann zu erklären. Sie versucht, ihr Handeln vor Walter zu rechtfertigen, die Schwere ihres Verbrechens zu leugnen und dadurch die Tatsachen zu verdrehen. So insistiert sie unter anderem darauf, immer gut zu den Gefangenen gewesen zu sein. Die auf der Ebene „Lager“ dargestellte Handlung bringt jedoch nach und nach ans Licht, dass Nichts an Lisas Verhalten gut war. Und dass sie darüber hinaus für den Tod von Marthas Verlobtem Tadeusz verantwortlich ist.

Dies zeigt sich in einer Episode, die sich um Martha und ihren Verlobten Tadeusz im Lager entspinnt: Denn als Martha und Tadeusz - nachdem sie sich jahrelang nicht gesehen hatten und sich gegenseitig tot glaubten - im Lager zufällig aufeinander treffen (4. Bild, 1 T. vor Z. 12) und Lisa ebenso zufällig Zeugin dieses Treffens wird, ${ }^{38}$ bietet sie den Liebenden scheinheilig an, auch weiterhin geheime Treffen zu unterstützen. Doch offensichtlich nur im Austausch für Gefälligkeiten. Tadeusz weigert sich jedoch daraufhin, das Angebot anzunehmen. Und obwohl es letztendlich seinen Tod bedeutet, erklärt er Lisa sehr offen, dass er nicht in ihrer Schuld stehen wolle (5. Bild, ab Z. 18). Als Lisa wiederum Martha von Tadeusz Weigerung, in ein Treffen einzuwilligen berichtet - um dadurch Marthas Glauben an Tadeusz' LIebe zu erschüttern -, hält Martha an ihrem Liebsten fest. Durch diese Haltung demonstrieren die Liebenden ihre unerschütterliche moralische Standhaftigkeit und Integrität.

Auf der Ebene „Überseedampfer“ sucht Lisa ihr Handeln verzweifelt vor Walter zu rechtfertigen und sich selbst aus der Verantwortung zu nehmen. Doch die wiederholte Konfrontation mit der unbekannten Passagierin zwingt Lisa letztendlich, sich und ihrem Ehemann einzugestehen, dass ihre verdrängten Erinnerungen verzerrt und unwahr sind. 


\section{Ein Hauptthema der Oper - die (r)echte Erinnerung}

Tatsächlich ist die Frage nach der Erinnerung - die Frage, was unter welchen Bedingungen wie und mit welchem Ergebnis erinnert werden kann und darf ein entscheidender Aspekt der Oper, dem besondere Aufmerksamkeit gebührt. So rückt schon das Epigraph, welches Weinberg in sein Partitur-Manuskript einfügte, den Vorgang des Erinnerns ins Zentrum: „Wenn das Echo ihrer Stimmen verhallt, gehen auch wir zugrunde" (Abbildung 1). ${ }^{39}$

AbBiLdung 1 Das Epigraph von Weinbergs Partitur-Manuskript

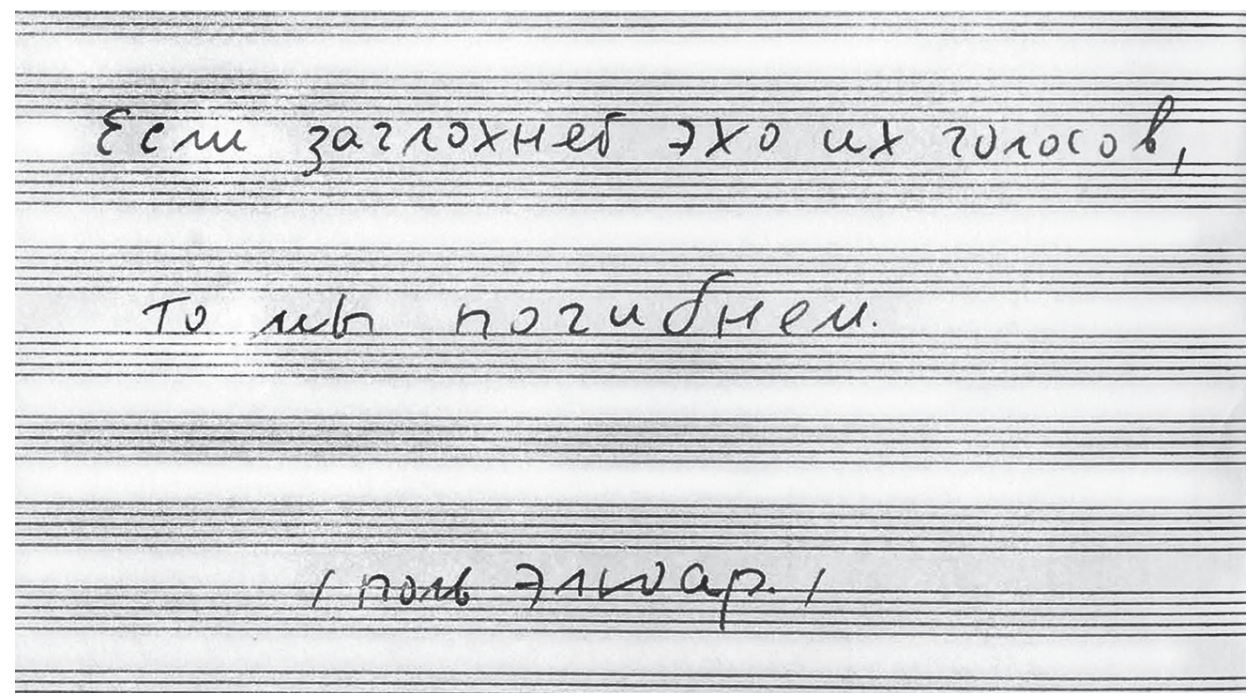

Dieses Zitat, das dem französischen Lyriker Paul Elouard zugeschrieben wird, erscheint nicht nur als Epigraph, sondern spielt auch im Epilog der Oper eine tragende Rolle. Denn dort singt Martha ihre finale Arie, in der sie den Wortlaut des Epigraphs zitiert (Epilog, ab Z. 4). Die Arie endet auch mit den Worten: „Ich werde euch, werde euch nie und nimmer vergessen...".40 So wird die Oper von einer Beschwörung des rechten Erinnerns und des Bewahrens textlich und musikalisch eingerahmt.

Auch die gesamte Konstruktion des Librettos, die Dramaturgie des Geschehens, basiert maßgeblich auf dem Akt des Erinnerns. So ist ein entscheidendes Moment der Handlung jener Moment, in dem Lisa auf dem Dampfer versucht, ihre quälenden Erinnerungen zu unterdrücken. Walter, der sich nach den ersten

39. Ich danke Peermusic Classical für die freundliche Genehmigung der Abdrucksrechte für diese Abbildung und der folgenden Notenbeispiele - V. M.

40. Weinberg, Die Passagierin, 28. 
Enthüllungen seiner Frau entsetzt zeigt, fragt Lisa wiederholt: „Hast Du wirklich alles gesagt? ${ }^{* 41}$ Doch Lisa windet sich, sucht nach Ausflüchten - und dies ist denn just der Punkt, in dem auch der Chor, wie bereits erwähnt, das erste Mal in die Handlung eingreift und Lisa drängt: „Na los, so sag's, so sag's, na los!‘42 Als Lisa daraufhin verzweifelt versucht, sich aus der Affäre zu ziehen (1. Bild, 8 T. nach Z. 7) und beteuert: „Alles, alles!“, weist der Chor sie zurecht und widerspricht: „Nein, nein, nein, nein, nein! Du hast nicht alles gesagt, nicht alles!“ (1. Bild ab T. 2 vor Z. 66).

Lisa insistiert, doch der Chor widerspricht erneut. Und nach seinen Worten „Dann werden jetzt einmal andere sprechen, sprechen, jetzt mögen andere sprechen!“ (1. Bild, ab T. 4 nach Z. 66) findet der Zeitsprung statt, der die Handlung erneut ins Lager und damit zu den Geschehnissen um die unbekannte „Passagierin“ führt. Der düstere, archaisch anmutende Gesang des Chores kontrastiert in dieser Szene musikalisch den schrillen Gesang Lisas, der mehr einem Schreien ähnelt. ${ }^{43}$

Auch auf der Ebene „Lager“ kommt dem Akt des Erinnerns eine bedeutende Rolle zu - wenngleich unter anderen Vorzeichen. Zunächst werden im dritten Bild des ersten Aktes verschiedene Mitgefangene als Figuren eingeführt. ${ }^{44}$ Yvette, ${ }^{45}$ eine junge Französin, Krzystina, eine Polin, ${ }^{46}$ Hannah, eine jüdische Frau, Vlasta, eine Tschechin und Katja, eine Russin. Jeder dieser Figuren wird ein Moment zugestanden, in dem eine Erinnerung oder das Anknüpfen an eine Erinnerung das Leiden im Lager erträglicher macht. Eine Erinnerung, die erlaubt, Verbindung zur verlorenen Heimat oder zu etwas Vertrautem herzustellen, wodurch innerhalb der Hölle von Auschwitz Augenblicke der Menschlichkeit aufflackern. So beispielsweise im Falle von Yvette gleich zu Beginn des 3. Bildes.

Yvette gehört zu einer Gruppe von Neuankömmlingen im Lager, und das junge Mädchen wird angesichts des Grauens von Entsetzen übermannt. Sie schreit vor Angst und ist außer sich. So ist Yvettes erster Gesangseinsatz (3. Bild, Z. 2) der verzweifelte Angstruf „Eine Hexe! eine Hexe!“ in der mehrfachen Wiederholung eines $\mathrm{a}^{2}$ im fortefortissimo, welches glissando abwärts rutscht. Als jedoch Krzystina, eine Gefangene, die sich schon länger im Lager befindet, die Neuankömmlinge nach ihrer Herkunft fragt, beruhigt sich die Szene. Yvette nennt ihren Namen und singt: „Ich soll ihn vergessen, das hat man mir geraten. ${ }^{“ 47}$ Die Musik jedoch kontrastiert diese Aufforderung zum Vergessen. Denn die musikalische Figur, in der die Gesangslinie der Yvette ausgestaltet ist, ist eine leichtfüßige und

41. Akt I, Bild 1; vgl. Weinberg, Die Passagierin, 11.

42. Weinberg, Die Passagierin, 11.

43. Lisas „Alles!“-Rufe erklingen in einer nach oben rückenden Figur von Achtel-Wechselnoten, die sich bis zum $f f$ auf eine Tonhöhe von $\mathrm{f}^{2}$-ges ${ }^{2}$ steigert.

44. Akt I, Bild 3; vgl. Weinberg, Die Passagierin, 13ff.

45. Die Schreibweise der Namen folgt der Übertragung im deutschsprachigen Libretto.

46. Die Figur der Bronka bleibt ohne explizite Zuweisung eines Herkunftsorts. Doch legen Notizen im Manuskript der Partitur nahe, dass auch sie aus Polen stammt.

47. Weinberg, Die Passagierin, 14. 
elegante, abwärtsführende Linie in Sekund-Wechselnoten, begleitet von einer getupften Achtelfigur aus pizzicato-Zweiklängen col legno in den Bratschen und Violinen. Die Musik illustriert demnach die französische Herkunft mit musikalischen Mitteln und kolportiert gleichzeitig in ihrer Verspieltheit die Jugend der Sängerin. Somit ist hier von Vergessen auch auf Ebene der Musik nichts zu hören. Zudem wird durch die plötzliche Intimität und klangliche Lieblichkeit der Szene auch musikalisch vermittelt, wie der Vorgang des Erinnerns Yvette beruhigt. So berichtet das Mädchen anschließend von ihrer Heimatstadt Dijon, von ihrer Mutter und ihrem Vater. ${ }^{48}$

Gänzlich anders verhält es sich indes bei der Figur der Russin Katja. Katja wird im 6. Bild (ab Z. 40) von ihren Mitgefangenen gebeten, von ihrer Heimat Russland zu berichten. Katja ist zunächst ein wenig ratlos und fragt: „Kann man denn davon erzählen?“ Doch Martha bittet sie erneut: „Dann singe ... irgendein altes Volkslied. Bitte!“ und Katja fügt sich: „Ein altes Lied? Welches nehm’ ich? Vielleicht dieses? Großmutter sang es häufig" (12 T. nach Z. 40). Sie hebt an zu singen (ab Z. 41) und der Gesang sticht aus dem Gesamtgefüge der Oper deutlich hervor. Denn Weinberg verzichtet an dieser Stelle auf jegliche Instrumentierung und lässt Katja ihre Arie unbegleitet singen. Das klagende und wehmütige Lied, das auch durch seine musikalische Isoliertheit einen verlorenen, verletzlichen Eindruck macht, wird jedoch nicht vollendet. Mitten im Singen bricht Katja ab (1 T. vor Z. 45) - da sie sich nicht mehr erinnern kann: „Weiter weiß ich nicht. Hab’s vergessen, vergessen. ${ }^{49}$ So wird es ausgerechnet der Figur der Russin Katja verwehrt, in der Erinnerung Zuflucht zu finden.

Bemerkenswert ist auch eine kleine musikalische Geste, die Weinberg vor allem im Kontext von Martha und Tadeusz verwendet. Es ist eine kleine Figur aus mehreren fallenden und ansteigenden Vorschlägen, die der Komponist (der generell spärlich mit Ornamentierungen arbeitete) vor Opus 97 in variierter Form vor allem in seinen Tuwim-Kompositionen verwendet hatte, und auch hier nur in bestimmten Kontexten. ${ }^{50}$ In Passažirka treten sie u. a. im 5. Bild auf, kurz bevor Tadeusz auf Lisa trifft. ${ }^{51}$ In der Szene selbst gibt Tadeusz Lisa endgültig zu verstehen, dass er auf ihr Angebot, mit ihr ,zusammenzuarbeiten' nicht einzugehen gedenkt. Dadurch demonstriert er seine moralische Überlegenheit, besiegelt jedoch gleichzeitig sein Schicksal. Eine strukturell ähnliche Verzierung verwendete Weinberg erstmals am Anfang des 4. Liedes seines (unveröffentlichten und unaufgeführten) Tuwim-Zyklus' Wspominenia [Erinnerungen] op. 62, den er bereits 1957/58 komponiert hatte. Der Text, den Weinberg in dem Lied

48. Ebd.

49. Ebd.

50. Ljudmila Nikitina, die erste sowjetische Wissenschaftlerin, die sich noch zu Lebzeiten Weinbergs eingehend mit dessen Werk beschäftigt hatte, nannte diese Ornamentierung das „Leitmotiv der Blumen“; Nikitina, Simfonii, 119.

51. Etwa im 5. Bild, 3 T. vor Ziffer 4. 
mit dem Titel Przypomnienie [Erinnerung] vertonte, ist eine hochgradig melancholische Meditation auf den Verlust der Heimat und den Verlust von Zugehörigkeit sowie auf ein Gefühl der Entfremdung von dem (fremden) Ort, der nun für sich in Anspruch nimmt, Heimat zu sein. Weiterhin taucht eine ganz ähnliche Ornamentierung im 2. und 5. Satz der 8. Symphonie op. 83 mit dem Titel Kwiaty polskie [Polnische Blumen] auf, die Weinberg bereits 1964 komponiert hatte (Notenbeispiel 1). Wichtig ist dabei zu wissen, dass auch die 8. Symphonie ein Werk ist, in dem Weinberg hauptsächlich Texte aus dem gleichnamigen Epos Tuwims verarbeitete. In diesem Werk beschäftigte sich der Dichter intensiv mit dem Verlust der Heimat und der Problematik des Daseins im Exil. ${ }^{52}$ Weiterhin verwendete Weinberg im 6. Satz der 8. Symphonie ein Gedicht Tuwims, in dem an die nachkommende Generation appelliert wird, sich stets der eigenen Wurzeln zu erinnern und sie niemals zu vergessen. ${ }^{53}$ Zwar ist die 8 . Symphonie eines der wenigen Tuwim-Werke, welches aufgeführt wurde, doch war dies u. a. dem Umstand zu verdanken, dass Weinberg (bzw. seine Übersetzerin) massiv in die russischen Übersetzungen der polnischen Texte eingriff und die polnischen Worte ,Jude“, ,jüdisch“ oder „Pole“, „polnisch“ in der Übersetzung durch ,neutrale“ Begriffe ersetzt wurden.

Indem Weinberg diese kleine, doch signifikante Ornamentierung nach Opus 62 und 84 nun in Passažirka erneut aufgriff, stellte er subtil eine Verbindung zwischen der Oper und den älteren Tuwim-Kompositionen her. Dem engsten Kreis von Feunden und Kollegen um Weinberg, die mit seiner Musik bestens vertraut waren, musste diese Verbindung aufgefallen sein. Nicht nur in musikalischer Hinsicht, sondern vor allem im Hinblick auf die Interpretation.

Die Oper endet mit dem Fazit, dass alle Dinge immer und vor allem wahrhaftig erinnert werden müssen. Diese Erkenntnis siegt über Lisa und ihre Versuche, sich durch verdrängte und verzerrte Erinnerungen selbst zu retten. Auch darin wird die Figur der Lisa und ihr Konzept der Erinnerung kontrastiert durch die Figur der Martha und die Form der wahrhaften Erinnerung, die ihr zu eigen ist. Weiterhin gehört Martha einer Sphäre an, in der Erinnerung Zugehörigkeit, Selbsterkenntnis und Stärke bedeutet. Eine Sphäre, die Lisa verschlossen bleibt. Darüber hinaus wird Lisa letztendlich durch die schiere Präsenz Marthas auf dem Dampfer gezwungen, sich den eigenen Erinnerungen und der Wahrheit zu stellen.

In der Sowjetunion jedoch, wo auch in poststalinistischen Zeiten nicht nur fortwährend die eigene Vergangenheit deformiert, sondern darüber hinaus auch

52. Vgl. dazu u. a. bei Marci Shore, Caviar and Ashes. A Warsaw Generation's Life and Death in Marxism, 1918-1968 (New Haven, CT - London: Yale University Press, 2006), 198, oder Elvira Grötzinger, „Das ,Judenkind“ und der , schreckliche Bürger‘. Ein Panoptikum der Zwischenkriegsjahre in Julian Tuwims Lyrik, in Juden und Judentum in Literatur und Film des slawischen Sprachraums. Die geniale Epoche, hrsg. von Peter Kosta u. a. (Wiesbaden: Harrassowitz, 1999), 155-172, hier 157.

53. Das Gedicht trägt den Titel „Lekcija“ [Unterrichtsstunde] und wurde von Tuwim bereits 1939 verfasst. 
NotenBeisPiel 1

Weinberg, op. 62, 4. Lied, T. 1-11
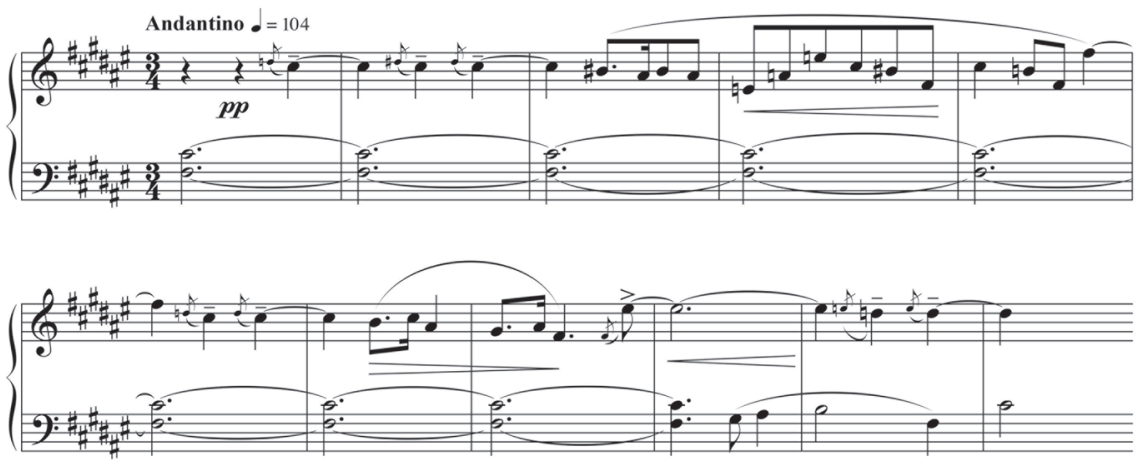

Weinberg, op. 97, 5. Bild, 3. T. vor Ziffer 4

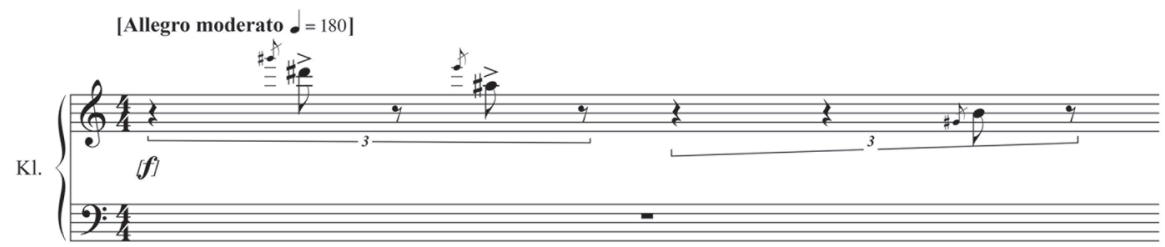

Weinberg, op. 83, 5. Satz, Anfang

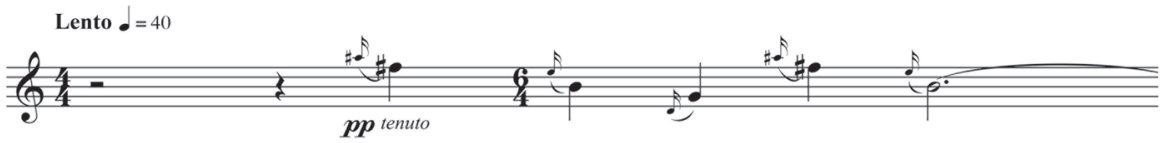

die Erinnerung an den Holocaust instrumentalisiert und verzerrt wurde, ${ }^{54}$ dürfte ein solches Resümee - noch dazu aufbereitet für die große Bühne - problematisch gewesen sein. Es muss als einer der Gründe dafür in Betracht gezogen werden, weshalb Passažirka nicht auf die Bühne gelangte. Umso mehr, als das Plädoyer für die wahrhafte Erinnerung von einem polnischen Juden in Töne gefasst worden war: dem Komponisten Mieczysław Weinberg.

54. Vgl. dazu u. a. Il'ja Al'tman: „Die Widerspiegelung der nationalsozialistischen Politik der Judenvernichtung in der sowjetischen Literatur und Publizistik (1940-1980)“, in „Zerstörer des Schweigens“. Formen künstlerischer Erinnerung an die nationalsozialistische Rassen- und Vernichtungspolitik in Osteuropa, hrsg. von Frank Günter u. a. (Köln u. a.: Böhlau, 2006), 17-32, v. a. 21-23. 
